\title{
Axillary Lymph Node Biopsy
}

National Cancer Institute

\section{Source}

National Cancer Institute. Axillary Lymph Node Biopsy. NCI Thesaurus. Code C62658.

Surgical removal of tissues from the lymph nodes located in the cavity beneath the junction of the arm and the thorax (armpit) so that a biopsy can be performed. 